\title{
Reviews
}

\section{Lung function testing assessment by impulse oscillometry in chronic lung disease}

\author{
Roxana Elena Cîrjaliu' ${ }^{1}$ Ioan Anton Arghir ${ }^{1,2} \neq$, \\ Enis Beitula ${ }^{1,2} \neq$, lonel Odagiu ${ }^{2,3} \neq$, Ileana Ion $^{2} \neq$, \\ Maria Nicolae ${ }^{1}$, Elena Danteș ${ }^{2,4}$, Oana Cristina \\ Arghir ${ }^{2,4}$ \\ \# Equal contribution with first and second author \\ 1 "St Andrew" Clinical County Emergency Hospital, \\ Constanța, Romania \\ 2 "Ovidius" University of Constanța, Constanța, Romania \\ ${ }^{3}$ Naval Medicine Center, Constanța, Romania \\ ${ }^{4}$ Clinical Hospital of Pneumology, Constanța, Romania
}

Corresponding author

loan Anton Arghir

ionut_arghir93@yahoo.com

\begin{abstract}
Impulse oscillometry (IOS) is a variant of forced oscillation technique described by Dubois 50 years ago, which allows us to measure the reactance of the airways and the resistance of the small and large airways during tidal breathing. It requires minimal patient cooperation from subjects who are unable to perform spirometry, like elders, children and patients with neurologic disorders. IOS can outline the diagnosis of obstructive airway disease, differentiate small airway obstruction from large airway obstruction. It is more sensitive than spirometry for peripheral airway disease in determining the severity of the disease, the exacerbations and evaluate the therapeutic response. Other applications include early evaluation of transplant rejection, cystic fibrosis, vocal cord disorder, bronchiectasis, hypersensitivity pneumonitis, obstructive sleep apnea.
\end{abstract}

Keywords: impulse oscillometry, lung function, obstructive airway disease

\section{Introduction}

Lung function is essential to monitoring several lung disorders, mostly obstructive airway diseases (OADs), conventionally diagnosed based on spirometry. Asthma and chronic obstructive pulmonary disease (COPD) are the most common OADs' entities, caused by smoking, occupational exposure, and indoor and outdoor pollution. Still, they can be secondary to alpha1antitrypsin deficiency (AAT) and tuberculosis (TB), as well. Impulse oscillometry (IOS) is an effort-independent non-invasive method of forced oscillation technique (FOT), a complementary way of spirometry, essential for revealing the early stages of obstructive lung diseases (OLDs) and for measuring the airway resistance at different bronchial tree' sites during spontaneous (tidal) breathing [1,2]. Dubois described FOT in 1956 by detecting the airway flow and pressure's disturbances inside the bronchial branches, expressed by physical parameters as resonant frequency (Fres), impedance, and peripheral airway resistance $\left(\mathrm{R}_{5}-\mathrm{R}_{20}\right)$ to airflow [3]. This ancient method is easily performed by children and elders. It does not require forced maneuvers of coordinated inhaling and exhaling or any closure of a valve in 
in connection to a mouthpiece.

Structural airways alterations in OLDs consist of progressive airway luminal narrowing, airway remodeling, subepithelial fibrosis of the airways wall, deposition of collagen inside the airway wall. Compared to hyperplasia and hypertrophy of smooth muscle cells in large airways are more evident in small airways causing irreversible airflow. In contrast, COPD being predominantly considered a small airway disease, there is more and more recognition of small airway disease as an essential phenotype of asthma $[1,4,5]$. The gold standard for the lung function's assessment of COPD patients is spirometry. However, in clinical practice, this method is not useful enough for early detection of airway disease in smokers, in the early stages of COPD, in small airway obstruction and dysfunction among uncontrolled asthmatic patients. Forced expiratory volume in the first seconds (FEV1) and maximal expiratory flow (MEF) are weak parameters in measuring the peripheral/small airway disease. IOS uses sound waves to measure respiratory mechanics, resistance, and reactance. IOS is a type of forced oscillation that combines "impulse-shaped" oscillations and "impulse pressures", being more sensitive than spirometry in detecting the earlier changes of lung function. And it is instrumental in differentiating the peripheral airway dysfunction from the large airway obstruction $[1,2]$.

The main advantage of IOS, in clinical practice, consistsinbeinga noninvasivemethod easy to perform, in all individuals from small children to geriatric patients, involving Tidal breathing, relaxation, and minimal cooperation, by measurements of different frequencies and variations of resistance and reactance to airway flow in the lung periphery $[1,6,7]$. The requirement of normal breathing does not require any effort from the subjects, inclusive for patients with breathing limitations and decreased inspiratory flow, with poor motor coordination and cognitive impairment, severely ill persons who are on ventilators, or underwent surgery. Oostveen E et al. presented a suggestive diagram of the forced oscillatory respiratory impedance measurement [8].

\section{The principle of the method pneumochromatograph}

To perform the test, the subject in a seated position and slightly extended head position, with a nose clip, is breathing normally through the mouthpiece of a pneumochromatograph, which has a pressure transducer for measuring the pressure and flow resistance and reactance. During the investigation, a nurse or a respiratory technician should firmly press the patient cheeks. FOT uses the oscillating sound signals of noise with different frequencies, generated by a loudspeaker, and transmitted sequentially to the peripheral airways [8]. These pressure sound waves are transferred to the subject's lung, causing airflow pressure changes in the airways according to the lung function's mechanical properties and airways resistance. Large airways reflect higher frequencies of the sound waves. Lower waves succeed in reaching the alveolar area and reflect, providing data about this pulmonary region [8].

Flow air recording shows the superposed sinusoidal waves in normal Tidal breathing. When sound waves overlap during tidal breathing, this results in a change in the flow. The straight line segment is inserted between the start and endpoints of the flow recordings due to each wave and considers it the baseline. After the baseline correction shows the separation of the respiratory component, the record shows a mixed-signal consisting of respiratory and sound wave components, and disturbances in the flow occur because of the superimposed waves. The same procedures apply to the recordings of pressure concerning time. On a time scale, these recordings have to be converted into the frequency scale to calculate further the parameters of interest. Fast Fourier transforms a mathematical technique to convert the time scale to a frequency scale. Respiratory input impedance (Zrs) is calculated as the ratio of the resulting pressure and flow changes due to the external pressure waves.

Flow recording is initially performed on normal tidal breathing. Afterward, flow is recorded when tidal breathing is superimposed by $5 \mathrm{~Hz}$ waves (d) and 20 $\mathrm{Hz}$ waves. When a pressure wave with frequency $5 \mathrm{~Hz}$ or $20 \mathrm{~Hz}$ is applied, changes in flow are recorded.

The main difference between FOT and IOS consists of the impulse's characteristics. In IOS, the signal is a mixture of sound waves and different frequencies, from 5 to $30 \mathrm{~Hz}$, transmitted sequentially along the bronchial tree down to the alveoli. Frequencies higher than $30 \mathrm{~Hz}$ can cause discomfort to the patient and must be avoided. In IOS, rather than sending the pressure waves of different frequencies sequentially, an impulse (that mathematically consists of all frequencies from $5 \mathrm{~Hz}$ to $30 \mathrm{~Hz}$ ) is sent into the airways. The impulse used during IOS examination can be a little forceful to the subject when compared to the gentler plain sinusoidal waves of FOT, and it may even change the lung mechanics. Still, the advantages overcome these limitations of the technique. First, with IOS, we can calculate the impedance at every 
frequency from 5 to 30 , whereas, in FOT, we can calculate only at the frequencies of the used waves. Second, the improved signal to noise ratio makes it a better tool for detecting regional abnormalities that have small effects on lung mechanics. Last but not least, this also decreases the duration of the test. Together, these diagnostic applications lead to increased efficiency in a PFT laboratory.

\section{IMPULSE OSCILLOMETRY PARAMETERS AND THEIR INTERPRETATION}

\section{Impedance}

Respiratory impedance represents the sum of all forces that oppose the generated impulse. It is measured, at any frequency, using the ratio between the pressure difference and flow changes, corresponding to that frequency. Depending on the region where the pressure is measured, the impedance varies. For example, the pressure gap between mouth level and the alveoli gives the impedance of the airways, and the difference between the mouth and pleural pressures provides the total impedance of the lung. In IOS, the pressure measured at the mouth level is referenced to the atmospheric pressure, which is the pressure outside the chest wall. This is defined as the respiratory system ( $\mathrm{Zrs}$ ), and it includes the in-phase (real) component, which is the resistive component (Rrs), and the out-of-phase (imaginary) component, which is a reactive component (Xrs). Rrs can be viewed as the energy dissipation and Xrs as energy storage. Since IOS measures input impedance, all chest wall abnormalities and skeletal muscles will also be reflected in the measurement.

\section{Resistance}

The resistance, derived from the impedance, includes $80 \%$ contributed by the resistance due to central airways, $20 \%$ by small peripheral airways $(<2$ $\mathrm{mm}$ in diameter) in adults $[1,9,10]$. In children, the contribution of small airways is higher than in adults. Lung tissue and chest wall resistance are usually negligible. Resistance values are considered normal within the range of $150 \%$ of the predicted values. In healthy people, resistance is independent of the frequency, compared to patients with central airway obstruction. The resistance at all frequency increases, while, in small airway obstruction, the resistance at lower frequencies increases. In the distal airways, the resistance at higher frequencies is unchanged, because waves do not reach this area. In children, this frequency dependency of resistance might be normal compared to adults, where it is a high resistance for lower frequencies impulse. A normal resistance for higher frequencies indicates small airway obstruction in adults.

\section{Reactance}

Unlike resistance, reactance is frequency-dependent and includes two components: the inertia of the air column to move (inertance) and the lung's capacitance. By definition, capacitance is negative in sign versus inertance, which is positive. Capacitance can be interpreted as a property that reflects the lungs' elasticity. Since the elastic properties of lungs majorly reside at the periphery, the capacitance component dominates at low frequencies. Total lung reactance is negative, whereas, at higher frequencies, the inertia of the air column in larger airways dominates, making the total reactance positive. The direction of change of reactance does not differentiate between obstructive or restrictive diseases. Similar to electric circuits, elastance or capacitance refers to the lung's energy return properties, not to stiffness during inflation. Therefore, in either lung fibrosis, or emphysema, or small airway disease, the reactance at lower frequencies would change, in the same direction, becoming even more negative (more harmful?).

\section{Resonant frequency}

Resonant frequency (fres) is the frequency at which the inertial properties of the airway and the capacitance of the lung periphery are equal, the frequency at which total reactance is zero. Fres cannot be attributed to a specific mechanical property of the lung. Where the capacitance component dominates, it can be used to separate low frequencies, from high frequencies, where the inertial component takes over. The normal value of fres in adults is $7-12 \mathrm{~Hz}$. In children, it is higher and increases with decreasing age. In obstructive and restrictive lung diseases, fres are above the normal range because reactance becomes more negative at low frequencies in emphysema and lung fibrosis.

\section{Area of reactance}

Area of reactance (AX) includes the area under the reactance curve from the lowest frequency to the fres, namely the area between the reactance curve and 
the negative side of the $\mathrm{X}$-axis, reflecting the elastic properties of the lung and increasing in small airways disease. $\mathrm{AX}$ is a single measurement that summarizes the above parameters in correlation with resistance at lower frequencies.

\section{Coherence}

Coherence is an important parameter used to determine the validity and quality of the test results because it reflects the reproducibility of the impedance measurements. Coherence takes values between 0 and 1. Ideally, it should be $>0.8$ at $5 \mathrm{~Hz}$ and $>0.9$ at $20 \mathrm{~Hz}$ to validate the measurements in adults. There are no standard values in children. Coherence can decrease if the technique is improper, such as irregular breathing, glottis closure, and swallowing.

\section{Reference values}

Normal values for the adult and pediatric population are essential for a straightforward interpretation of the test. Studies to determine reference values in adults are very few. In children, the resistance and frequency of resonance decrease with increasing height and age, whereas reactance increases. Height and age are identified as the critical covariates and significant predictors of R5 and X5 [1,11,12]. The relationship between reactance and height is linear, while between resistance and height is mostly exponential. Shiota et al. found that predictive equations with significant differences in smokers and nonsmokers and no differences by gender in the Japanese population $[1,11]$. There is no predictive equation for the Romanian healthy population. Before obtaining a specific equation, data from other Caucasian populations might be used [1]. As for monitoring the evolution of lung disease, the interindividual differences in intra-individual changes, in time, can provide accurate enough data to sustain the improvement or the aggravation of the functional lung status.

\section{Role of IOS in Asthma}

IOS has been used to study several lung diseases, including asthma, monitoring the progress of diseases. The diagnosis of asthma includes a demonstration of reversible obstruction and bronchial hyperresponsiveness [13,14]. IOS has been demonstrated to be sensitive and accurate than spirometry revealing more elevated R5, AX, fres compare to healthy patients, where $\mathrm{X} 5$ is more negative $[15,16]$. Shi et al. compared baseline spirometry and IOS parameters between asthmatic children, subsequently presenting with exacerbations and children with controlled asthma [17].

Gonem et al. showed that impedance measured over time could differentiate between the frequent exacerbators from the less frequent exacerbators among asthmatic patients [18]. Studies also showed significant improvement of all IOS parameters in asthmatics, after three months of inhaled corticosteroids (ICS) administration, with or without a combination with long-acting beta-agonist (LABA). A decrease of R5 with $30-35 \%$ suggests a positive bronchodilator response [13]. IOS can be used to determine the early obstruction of the small airways, even when spirometry is normal [14]. In bronchoprovocation testing, an increase in R5, fres, and AX and a decrease in the X5 was observed. A 20\% decrease in FEV1 is shown to be equivalent to a $50 \%$ decrease in X5 [19]. Schulze et al. showed that IOS has a better performance identifying the bronchial hyperreactivity at lower doses of methacholine than the dose inducing a 20\% decrease of FEV1 from its initial value [20].

\section{Role of IOS in bronchitis}

The Tucson Children's Respiratory Study analyzed 130 participants with a personal medical history of bronchitis since the age of 32. IOS and spirometry parameters were regressed on height, weight, gender, and ethnicity. Participants with bronchitis had significantly elevated resistance at 5,10 , and $15 \mathrm{~Hz}$ and decreased reactance compare to healthy subjects [21].

\section{Role of IOS in recurrent wheezing}

IOS assessment of lung function in 115 recurrent wheezers, aged between 2-6 years, $65.2 \%$ males, showed R5-R20\% levels > 14.4 for predicting a positive modified asthma predictive index (mAPI) in $55.6 \%$ of children, identifying those with a high risk of asthma [22].

\section{Role of IOS in COPD}

In COPD patients, IOS's reactive parameters are better correlated with lung function than resistance [23]. Over time, changes in X5 were proposed for monitoring the disease [23]. Frantz et al. reported that, despite normal spirometry, subjects with symptoms of COPD have higher pulmonary resistance 
resistance and lower pulmonary reactance, indicating IOS as a more sensitive technique in detecting the subtle changes in the lung function in early COPD [24]. Peripheral airway resistance, expressed as frequency, dependent heterogeneity between $5 \mathrm{~Hz}$ and $20 \mathrm{~Hz}$ (R5R20), and peripheral airway compliance as the area under the reactance curve (AX), are affected in COPD. R5-R20\% and AX improve in response to long-acting bronchodilators [25]. The fact that spirometry is a diagnostic standard for COPD complicates matters. Since the diagnosis of COPD is based on spirometry, it is challenging to compare IOS. Still, there are some COPD studies to prove the usefulness of IOS in monitoring the disease progress. To define better Predictive Surrogate Endpoints Evaluation for COPD, in a large cohort of 2,609 COPD subjects, the IOS was determined at baseline [26]. COPD was associated with increased R5, R5 - R20, and reactance. However, $5-10 \%$ of smokers, older and heavy smokers, had "abnormal" IOS, possibly reflecting an earlier stage of COPD, undiagnosed by normal spirometry (lower values of FEV- 1 , but still in the normal range). This outcome has been confirmed by other researchers who report healthy smokers often have abnormal IOS compared to nonsmokers [27]. IOS is more sensitive at predicting future exacerbations of COPD. AX might also be considered a useful screening tool in the early stage of COPD or monitoring long term decline of lung function [28]. Another study showed improvement of R5 and AX in patients, who received COPD triple combination therapy compared to tiotropium monotherapy [29].

\section{Role of IOS in cystic fibrosis}

Cystic fibrosis (CF) is a multisystem disease with high rates of morbidity and mortality. Spirometry is the primary method used in the evaluation of $C F$ and the therapeutic response. IOS can reveal increased resistance and AX during CF exacerbations [30].

\section{Role of IOS in Hypersensitivity pneumonitis}

Hypersensitivity pneumonitis (HP) is a group of inflammatory interstitial lung diseases caused by hypersensitivity immune reactions to the inhalation or ingestion of various antigens like fungal, bacterial, animal protein, or chemical sources, including multiple occupational exposures [31]. In a study of 20 patients with HP, IOS showed elevated AX, at baseline, with improvement after administration of, particularly, azathioprine and prednisone. Lung volumes were also improved, but there was no influence on gas exchange values [32].

\section{The role of IOS in monitoring lung transplant}

IOS can be used to monitor patients with a lung transplant. A study involving 25 patients showed, in $88 \%$ of the cases, increased R5 and AX, indicating peripheral airway obstruction. IOS can be useful in evaluating early transplant rejection [33].

\section{The role of IOS in Bronchiectasis}

Bronchiectasis is a chronic respiratory disease involving recurrent bacterial airway infections, inflammation, and destruction, mostly related to post tuberculosis underlying lung disease [34].

Higher levels of IOS parameters are associated with Pseudomonas aeruginosa infection, inhomogeneity, and cystic bronchiectasis. All IOS parameters, except R5, were correlated with the duration of bronchiectasis symptoms, number of lobes with bronchiectasis and total scores of high-resolution computed tomography (HRCT), compare to increased frequency dependence (AX), which was associated with lower HRCT scores $(\leq 5)[35]$.

\section{The role of IOS in OSA}

IOS is useful in assessing patients with obstructive sleep apnea (OSA), revealing higher R5 and lower Xrs compared to healthy patients. The X5 parameter is associated with the severity of OSA, according to Apnea-Hypopnea Index (AHI). The severity of OSA (AHI) correlated with R20, R5, and R5-R20, a negative relationship was demonstrated between the level of average night saturation and R5-R20 and R20 [26]. A strong correlation between X5 and OSA severity suggests that a narrowing of the upper airways and abnormal increased lung elastic recoil are incriminated in OSA. IOS may have potential as a screening tool for OSA diagnosis as it can detect upper airways stenosis or patency in OSA patients [36]. A study showed significantly higher R20\% value in OSA patients than COPD patients, higher R5-R20\% values compared to the control group, and $\mathrm{X} 5$, in the supine position, as the best outcome for predicting OSA [37].

\section{Other applications of IOS}

IOS may also be useful in upper airway obstruction [38], vocal cord dysfunction [39], and musculoskeletal 
thoracic abnormalities [40]. Regarding the occupational setting, impedance measurements alongside spirometry can be used in evaluating subjects exposed to environmental irritants [41]. Numerous studies explored the role of IOS in early detection and characterization of respiratory dysfunction secondary to massive exposure to dust and smoke, the acute reactive airways syndrome, a severe occupational disease [42].

\section{Limitations}

Although IOS is useful, very easy to perform in elderly subjects, and small children, it has some limitations. It isn't easy to compare measurements taken by different devices. Intra-individual variability for all IOS parameters is higher than FEV1 $(\sim 10 \%$ vs. $\sim 5 \%)$, but it is acceptable in clinical practice. Notably, IOS parameters are less variable than other spirometry parameters, such as $\mathrm{FEF}_{50}(\sim 20 \%-30 \%)$. The use of IOS parameters in restrictive diseases is uncertain. Rrs and Xrs are volume-dependent parameters. Concerning the bronchodilator responses, there is a difference between IOS values and spirometry response. Most research on IOS has been carried out on relatively small groups of patients [43]. However, given its importance, IOS can be a part of the routine pulmonary examination. Further technological advances that reduce the form factor, making it inexpensive and portable and novel analytical methods for ease of diagnosis are on the horizon. Such developments would make IOS a viable alternative to spirometry in Pneumology and Occupational Medicine's routine clinical practice.

\section{Conclusions}

IOS may be more sensitive than spirometry in early detecting small airways' abnormalities. It is an advantageous, safe, non-invasive method for measuring the lung's mechanical properties, helping the diagnosis of obstructive lung diseases, including obstructive sleep apnea, and monitoring the progress of obstruction in small airways. It is also a better tool to predict early COPD, uncontrolled asthma, and exacerbations. However, since IOS is better for detecting small airway disease, in the future, it could be considered an investigation required to evaluate occupational OLDs, mostly when spirometry is normal. The main limitation is the lack of reference values and extensive evaluation of different disease conditions, including occupational exposure.
However, for a dedicated laboratory, these are not limitations of more than the technique itself and could be adopted widely with more thoroughly conducted studies.

\section{No financial support and sponsorship. There are no conflicts of interest.}

\section{References}

1. Desiraju K, Agrawal A. Impulse oscillometry: The state-of-art for lung function testing. Lung India. 2016; 33(4): 410-416.

2. Su ZQ, Guan WJ, Li SY, Ding M, Chen Y, Jiang M et al. Significances of spirometry and impulse oscillometry for detecting small airway disorders assessed with endobronchial optical coherence tomography in COPD. Int J Chron Obstruct Pulmon Dis. 2018;13:3031-3044.

3. Dubois AB, Botelho SY, Comroe JH. A new method for measuring airway resistance in man using a body plethysmograph: Values in normal subjects and in patients with respiratory disease. J Clin Invest. 1956; 35: 327-35.

4. Hogg JC, Chu F, Utokaparch S, Woods R, Elliott WM, Buzatu L, et al. The nature of small-airway obstruction in chronic obstructive pulmonary disease. N Engl J Med. 2004; 350: 2645-2653.

5. Contoli M, Bousquet J, Fabbri LM, Magnussen H, Rabe KF, Siafakas $\mathrm{NM}$, et al. The small airways and distal lung compartment in asthma and COPD: A time for reappraisal. Allergy. 2010; 65:141-51.

6. Smith HJ, Reinhold P, Goldman MD. Forced oscillation technique and impulse oscillometry. European Respiratory Monograph. Ch. 5. In: Gosselink R, Stam H, editors. Lung Function Testing. UK: European Respiratory Society Publications; 2005; 72-105.

7. Bickel S, Popler J, Lesnick B, Eid N. Impulse oscillometry: Interpretation and practical applications. Chest. 2014; 146: 841-847. 8. Oostveen E, MacLeod D, Lorino H, Farre R, Hantos Z, Desager K, et al. The forced oscillation technique in clinical practice: methodology, recommendations and future developments. Eur Respir J. 2003; 22: 1026-41.

9. Hogg JC, Williams J, Richardson JB, Macklem PT, Thurlbeck WM. Age as a factor in the distribution of lower-airway conductance and in the pathologic anatomy of obstructive lung disease. N Engl J Med. 1970; 282:1283-1287.

10. Mead J. The lung's “quiet zone” N Engl J Med. 1970; 282:13181319.

11. Shiota S, Katoh M, Fujii M, Aoki S, Matsuoka R, Fukuchi Y. Predictive equations and the reliability of the impulse oscillatory system in Japanese adult subjects. Respirology. 2005; 10: 310-315.

12. Newbury W, Crockett A, Newbury J. A pilot study to evaluate Australian predictive equations for the impulse oscillometry system. Respirology. 2008; 13:1070-5.

13. Song TW, Kim KW, Kim ES, Park JW, Sohn MH, Kim KE. Utility of impulse oscillometry in young children with asthma. Pediatr Allergy Immunol. 2008;19:763-768.

14. Marotta A, Klinnert MD, Price MR, Larsen GL, Liu AH. Impulse oscillometry provides an effective measure of lung dysfunction in 4-year-old children at risk for persistent asthma. J Allergy Clin Immunol. 2003;112:317-322.

15. Kim HY, Shin YH, Jung da W, Jee HM, Park HW, Han MY. Resistance and reactance in oscillation lung function refl ect basal lung function and bronchial hyperresponsiveness respectively. Respirology. 2009; 14(7):1035-1041.

16. Qi GS, Zhou ZC, Gu WC, Xi F, Wu H, Yang WL, et al. Detection of the airway obstruction stage in asthma using impulse oscillometry system . J Asthma. 2013; 50(1): 45-51.

17. Shi Y, Aledia AS, Galant SP, George SC. Peripheral airway 
impairment measured by oscillometry predicts loss of asthma control in children. J Allergy Clin Immunol. 2013;131:718-723.

18. Gonem S, Umar I, Burke D, Desai D, Corkill S, Owers-Bradley J, et al. Airway impedance entropy and exacerbations in severe asthma. Eur Respir J. 2012;40:1156-1163.

19. Bailly C, Crenesse D, Albertini M. Evaluation of impulse oscillometry during bronchial challenge testing in children. Pediatr Pulmonol. 2011;46:1209-1214.

20. Schulze J, Smith HJ, Fuchs J, Herrmann E, Dressler M, Rose MA, et al. Methacholine challenge in young children as evaluated by spirometry and impulse oscillometry. Respir Med. 2012;106:627634.

21. Asseri A, Debra Stern Stefano Guerra Anne Wright Fernando Martinez; and Wayne Morgan University of Arizona, Tucson, AZ, Impulse Oscillometry Compared to Spirometry in Adults With a History of Bronchitis, Chest 2016;150(4, suppl):1113A

22. Arikoglua T, Batmaz SB, Yildirim DD, Tezol Ö, Bozlu G, Kuyucu $S$. Asthma predictive index in relation to respiratory mechanics by impulse oscillometry in recurrent wheezers, Allergol Immunopathol (Madr). 2018;46(2):190-195.

23. Gong SG, Yang WL, Zheng W, Liu JM. Evaluation of respiratory impedance in patients with chronic obstructive pulmonary disease by an impulse oscillation system. Mol Med Rep. 2014;10:2694-7200. 24. Frantz S, Nihlén U, Dencker M, Engström G, Löfdahl CG, Wollmer P. Impulse oscillometry may be of value in detecting early manifestations of COPD. Respir Med. 2012;106:1116-1123.

25. Komarow HD, Myles IA, Uzzaman A, Metcalfe DD. Impulse oscillometry in the evaluation of diseases of the airways in children. Ann Allergy Asthma Immunol. 2011;106:191-199

26. Crim C, Celli B, Edwards LD, Wouters E, Coxson HO, Tal-Singer $\mathrm{R}$, et al. Respiratory system impedance with impulse oscillometry in healthy and COPD subjects: ECLIPSE baseline results. Respir Med. 2011;105:1069-78.

27. Shinke H, Yamamoto M, Hazeki N, Kotani Y, Kobayashi K, Nishimura Y. Visualized changes in respiratory resistance and reactance along a time axis in smokers: A cross-sectional study. Respir Investig. 2013;51:166-74.

28. Manoharan AW, Lipworth J, Ibrahim I, Lipworth B. Small Airways Dysfucntion Is Associated with Poorer Control in Asthmatic Patients with a Preserved FEV1 Chest 2014;44(suppl 58);3406

29. Mineshita M, Shikama Y, Nakajima H, Nishihira R, Komatsu M, Kunota M, et. al. The application of impulse oscillation system for the evaluation of treatment effects in patients with COPD. Respiratory Physiology and Neurobiology, 2014; 202:1-5.

30. Sakarya A, Uyan ZS, Baydemir C, Anik Y, Erdem E, Gokdemir Y, et al. Evaluation of children with cystic fibrosis by impulse oscillometry when stable and at exacerbation. Pediatr Pneumol. 2016;51(11):11511158.

31. Kaltreider HB. Hypersensitivity Pneumonitis. West J. Med. 1993;159:570-578.

32. Guerrero Zúñiga S, Sánchez Hernández J, Mateos Toledo H, Mejía Ávila M, Gochicoa-Rangel L, Miguel Reyes JL, et al. Small airway dysfunction in chronic hypersensitivity pneumonitis. Respirology. 2017;22(8):1637-1642.

33. Ochman M, Wojarski J, Wiórek A, Slezak W, Maruszewski M, Karolak W, et al. Usefulness of the impulse oscillometry system in graft function monitoring in lung transplant recipients. Transplantation Proceedings. 2018;50(7):2070-2074.

34. Barker AF. Bronchiectasis. N Engl J Med 2002;346:1383-1393.

35. Guan WJ, Gao YH, Xu G, Lin ZY, Tang Y, Li HM, et al. Impulse Oscillometry in Adults with Bronchiectasis. Ann Am Thorac Soc. 2015:12(5):657-667

36. Abdeyrim A, Li N, Shao L, Heizhati M, Wang Y, Yao X, et al. What can impulse oscillometry and pulmonary function testing tell us about obstructive sleep apnea: a case-control observational study? Sleep Breath. 2016;20(1):61-68.

37. Huiguo L, Wang N, Jianping Z, Shengdao X, Yongjian X, Zhenxiang $Z$. The diagnosis value and its implication of impulse oscillometry in obstructive sleep apnea syndrome patients. J Tongji Med Univ. 2000;20(4):280-282.

38. Verbanck S, de Keukeleire T, Schuermans D, Meysman M, Vincken W, Thompson B. Detecting upper airway obstruction in patients with tracheal stenosis. J Appl Physiol (1985). 2010;109(1):47-52.

39. Komarow HD, Young M, Nelson C, Metcalfe DD. Vocal cord dysfunction as demonstrated by impulse oscillometry. J Allergy Clin Immunol Pract. 2013;1(4):387- 393.

40. van Noord JA, Clément J, Cauberghs M, Mertens I, Van de Woestijne KP, Demedts M. Total respiratory resistance and reactance in patients with diffuse interstitial lung disease. Eur Respir J. 1989;2(9):846-852.

41. Morris MJ, Dodson DW, Lucero PF, et al. Study of active duty military for pulmonary disease related to environmental deployment exposures (STAMPEDE). Am J Respir Crit Care Med. 2014;190(1):7784.

42. Mauer MP, Cummings KR. Impulse oscillometry and respiratory symptoms in World Trade Center responders, 6 years post-9/11. Lung. 2010;188(2):107-113.

43. Bednarek M, Grabicki M, Piorunek T, Batura-Gabryel H. Current place of impulse oscillometry in the assessment of pulmonary diseases. Respir Med. 2020;170: 105952. 